\title{
COVID-19 in Children with Asthma
}

\author{
Grigorios Chatziparasidis $^{1}$ (1) $\cdot$ Ahmad Kantar $^{2}$
}

Received: 3 November 2020 / Accepted: 8 January 2021 / Published online: 26 January 2021

(c) The Author(s), under exclusive licence to Springer Science+Business Media, LLC part of Springer Nature 2021

\begin{abstract}
Severe acute respiratory syndrome coronavirus-2 (SARS-CoV-2) infects both children and adults but epidemiological and clinical data demonstrate that children are less likely to have a severe disease course or die. Furthermore, asthmatic children show less severe disease manifestations when infected with SARS-CoV-2 comparing to adults. This review focuses on SARS$\mathrm{CoV}-2$ and childhood asthma interaction and aims at summarizing the current knowledge of the potential mechanisms that ameliorate disease symptomatology in asthmatic children.
\end{abstract}

Keywords Asthma $\cdot$ Children $\cdot$ SARS-CoV-2 $\cdot$ COVID-19

\section{Introduction}

The end of 2019 was the beginning of an unprecedented time for humanity. An outbreak of the coronavirus disease (COVID-19), which is caused by the severe acute respiratory syndrome coronavirus-2 (SARS-CoV-2), emerged in the city of Wuhan, China [1]. In March 2020, the World Health Organization (WHO) declared COVID-19 to be a global pandemic [2].

SARS-CoV-2 is the newest member of the coronavirus family. Coronaviruses cause various human diseases involving the respiratory system, nervous system, and gastrointestinal tract. These viruses preferentially target the nose and lungs, leading to diseases such as the common cold, bronchitis, and pneumonia [3]. They represent the second most frequent cause of symptoms of the common cold in humans [4].

Coronaviruses are "spiky invaders" in that they use a spike protein $(\mathrm{S})$ to attach to human cell membranes, and the entire process is activated by specific cell enzymes. SARS$\mathrm{CoV}-2$ is more infectious than members of this family of viruses due to its unique spike protein activation by a host

Grigorios Chatziparasidis

gchatziparasidis@gmail.com

1 Primary Cilia Dyskinesia Unit, School of Medicine, University of Thessaly, Thessaly, Greece

2 Pediatric Asthma and Cough Centre, Instituti Ospedalieri Bergamaschi, University and Research Hospitals, Bergamo, Italy cell enzyme called furin. Furin resides in multiple human tissues, including the lungs, liver, and gastrointestinal tract, thus enabling the virus to attack multiple organs simultaneously. Furthermore, furin activation promotes the stability and transmission of the virus. These properties have enabled the rapid spread of SARS-CoV-2 worldwide [5].

With the virus being so easily transmitted, effects on the pediatric community were inevitable, and the very first reported case was encountered in January 2020 in a 10-yearold boy who contracted the virus in Wuhan [6]. Since then, a multitude of pediatric cases have been reported globally. To date, the data reveal that the course of the disease is milder in children than adults, but it is unclear what the role of type- 2 inflammation is in the outcome of asthmatic children infected by SARS-CoV-2.

\section{Why Asthmatic Children were Initially Thought to have a Gloomy Outcome During the Pandemic}

COVID-19 has resulted in uncharted challenges for clinicians, who have had to act and prescribe treatments based on limited data. This is why the pediatric community has feared about the future of asthmatic children infected by the virus. These fears were based on the following data:

1. The pathophysiology of asthma includes mucus hypersecretion, damaged epithelium, and airway obstruction. Additionally, the inhaled corticosteroids that are the cor- 
nerstone of asthma treatment promote viral replication, delay viral clearance, and result in local immunosuppression, thus theoretically creating the perfect host for SARS-CoV-2 [7].

2. A 2002 Lancet study emphasized that people with asthma have an increased risk of more severe outcomes when infected with common cold viruses. As previously mentioned, coronaviruses are the second most common cause of colds in humans [8]. Other studies underscore the direct relationship between virus-induced exacerbation severity and the degree of lack of control in asthmatics [9].

3. At the tissue level, the innate anti-viral immune response is delayed and deficient in asthmatics when they are infected with respiratory viruses. Particularly, they mount a suboptimal response of interferon (IFN)- $\alpha$, IFN- $\beta$, and IFN- $\lambda$, of which the latter is associated with increased severity of asthma exacerbation [10-12].

Thus, it would seem prudent for physicians to include pediatric asthma under the umbrella of high risk factors for severe COVID-19 outcomes. Fortunately, however, the current literature does not support this view. A systematic review by Castro-Rodriguez [13] found only two studies alleging that recurrent wheezing or asthma is a potential risk factor for COVID-19 in children. More importantly, in large epidemiological studies, asthma was not included among the risk factors for either SARS-CoV-2 infection or COVID-19 severity [14].

Recent large studies focusing on the course of asthmatic children during the pandemic revealed that they are not disproportionately affected by the virus, and surprisingly, they appear to have improved outcomes [15]. This was attributed to reduced exposure to asthma triggers, increased steroid prescriptions, and better treatment adherence during the pandemic. Another possible contributing factor is the timely adaptation of the clinical services to the pandemic by replacing physical appointments with virtual ones [16].

\section{Clinical Manifestations of COVID-19 in Children}

A review study examined 1124 pediatric cases and stratified COVID-19 symptoms based on their severity and frequency. According to the study, once infected with SARS-CoV-2, a child might follow one of the following clinical patterns [17]:

1. Asymptomatic infection ( $14.2 \%$ of cases). These children have no clinical symptoms, and the infection is confirmed by a positive nucleic acid test.
2. Mild disease (36.3\% of cases). A child develops the symptoms of an illness of the upper respiratory tract, and the most common is fever. Other symptoms include cough, myalgia, sore throat, nasal catarrh, and sneezing. Fewer children might present with gastrointestinal symptoms, such as abdominal pain, nausea, emesis, and diarrhea. The results of chest auscultation are unremarkable.

3. Moderate disease ( $46 \%$ of cases). This group of children presents with pneumonia.

4. Severe disease $(2.1 \%$ of cases $)$. The initial manifestations might be a combination of symptoms of the respiratory and digestive tracts, followed by dyspnea and central cyanosis within a week. Oxygen desaturation below $92 \%$ is a striking feature.

5. Critical cases ( $1.2 \%$ of cases). A minority of infected children progress to acute respiratory distress syndrome with or without multiple organ dysfunction.

Concordantly, a review paper emphasizes that the majority of children with COVID-19 either remain asymptomatic or develop a less severe phenotype. Notwithstanding, some infants might be seriously ill, and some older children might develop a multisystem inflammatory syndrome necessitating admission to intensive care. Three deaths were also reported, and the authors mentioned that "although most pediatric COVID-19 patients were not severe, a serious COVID-19 illness could result in severe outcomes including an ICU admission and even death in children" [18].

\section{Why is SARS-CoV-2 Milder in Children?}

Children seem to elude severe COVID-19 manifestations and face a better outlook than adults. This is likely due to the way that SARS-CoV-2 invades human cells. The virus's S protein binds the host cell's angiotensin converting enzyme (ACE) 2 receptor, facilitating its attachment to host cell membranes [19]. Thus, it seems that the renin angiotensin system, which plays a key role in the regulation of arterial blood pressure, is actively involved in the viral attachment and activation of an inflammatory process in multiple organ systems, including the lungs, kidneys, heart, and brain. ACE converts angiotensin (AT)-I into AT-II, which in turn is converted to AT by activated ACE2. Surprisingly, AT-II can induce pro- inflammatory actions in these organs by binding to the AT1 receptor, which seems to occur in COVID-19 cases [20-22].

In humans, the nose is the first site of viral invasion into the body by the SARS-CoV-2 virus. After penetrating the nose, the virus attaches to ACE2 receptors, kicking off a cascade of events that lead to COVID-19. Children may be less likely to experience this first step for two possible reasons: 
first, children have lower expression of ACE2 receptors in their nasal epithelium and lower airways than adults [23] as the expression in the respiratory tract increases with age $[24,25]$. Second, children are more likely to have acquired immunity to common coronaviruses since they are more commonly exposed to a wide variety of pathogens. These include the coronaviruses that cause mild illnesses of the upper respiratory tract, such as the common cold, or more severe illnesses such as bronchiolitis and croup. These illnesses could potentially be associated with the downregulation of ACE2 expression in respiratory epithelial cells and antibodies that potentially cross-react to SARS-CoV-2 [23, 26, 27].

Therefore, in children carrying ACE2 downregulating viruses (e.g., human coronavirus NLg3), this decreased expression might interfere with the replication of SARSCoV-2. This phenomenon, where one virus interferes with the replication of a second virus, is called viral interference [28]. Additionally, children experience higher mucosal colonization by viruses and bacteria, resulting in interactions and competition, which limits the colonization and growth of SARS-CoV-2 [27].

Despite these deterrents, SARS-CoV-2 still infects children by sticking its spike into the respiratory epithelial cells via ACE2, thus entering the cell. Cell entry is facilitated by proteolytic cleavage involving two proteases: transmembrane protease serine 2 (TMPRSS2) and cathepsin L (CTSL) [29, 30]. SARS-CoV-1 utilizes a similar method to infect human respiratory cells, and it has been shown that TMPRSS2 activity reduces viral recognition by human defense mechanisms. However, whether the same applies to the SARSCoV-2 virus is still unclear [31].

From the nose, the SARS-CoV-2 virus travels to the lower airways and finally the alveoli, where it attaches to ACE2 receptors located on both type I and II alveolar cells, which also express TMPRSS2. There are reports that children have fewer of these molecules in their alveolar cells than adults [24]. Most importantly, cells expressing both ACE2 and TMPRSS2 are quite rare in children but increase with age. These so-called "double-positive cells" overexpress interleukins IL-6 and IL-6R, leading to overactivation of the immune system and the possible initiation of the cytokine storm that occurs in adults [25].

These data cast light on why children are not only less affected by COVID-19 but are also less likely to progress to acute respiratory distress syndrome (ARDS). Of 2143 Chinese pediatric patients with confirmed $(n=731)$ or suspected $(n=1412)$ COVID-19, more than half had mild disease, and $<1 \%$ developed severe or critical symptoms [32]. A hospital-based US study involving 60 adult and 65 "pediatric" COVID-19 patients (aged $<24$ years) attempted to explain the differences in the disease outcome. Of the pediatric patients, 20 had novel multisystem inflammatory syndrome (MISC), which occurs in a minority of children infected with COVID-19. The symptoms include fever, laboratory evidence of inflammation, serological evidence of recent SARS-CoV-2 infection, and any combination of cardiac, renal, hematological, dermatological, neurological, gastrointestinal, or respiratory disease [33, 34].

Twenty-two adults (37\%) and five children (8\%) required mechanical ventilation, and no deaths occurred among the MISC pediatric patients. The authors speculated that children with COVID-19 have better outcomes because of their stronger innate immunity (higher lymphocyte count, higher absolute numbers of $\mathrm{T}$ and $\mathrm{B}$ cells, and more natural killer cells), which is capable of producing higher levels of cytokines, particularly IL-17A. This cytokine appears essential in protecting children from developing ARDS. Humoral immunity is recruited in both adult and child patients, but at different strengths, and both mount an antibody response against the $\mathrm{S}$ protein (including neutralizing antibodies that block cell invasion) (Tables 1 and 2).

Counterintuitively, the levels of neutralizing antibodies were higher in adult patients who needed ventilation assistance or died of the disease than in patients who recovered, but the levels were notably higher than those in pediatric patients. This indicates that the over-vigorous adaptive immune response reported in adult patients can lead to an over-inflammatory state and eventually ARDS or death [35]. Childhood is also characterized by a lower prevalence of co-morbidities that are considered risk factors for a severe course of COVID-19, such as arterial hypertension, diabetes mellitus, and chronic heart, lung, and kidney diseases [36].

\section{Do Asthmatic Children Perform Differently When Infected by SARS-CoV-2?}

The exact way in which SARS-CoV-19 behaves in asthmatic children seems to be different than in asthmatic adults. Data from the UK associate severe asthma with worse outcomes in adult COVID-19 cases [37]. A potential answer for the differing severity between asthmatic adults and asthmatic children might lie in the asthmatic phenotype. The incidence of allergic asthma peaks in early childhood and steadily decreases with age. Non-allergic asthma has a low prevalence in childhood and peaks in late adulthood [38].

We previously mentioned that ACE2 and TMPRSS2 expression mediates SARS-CoV-2 infection of host lung cells. Notably, the expression of ACE2 and TMPRSS2 is higher in patients with diabetes, who are at a higher risk of mortality and complications from COVID-19 [39]. One study reported that asthma with allergic sensitization is characterized by lower ACE2 expression in children's nasal epithelia, but not in non-allergic asthmatic children [40]. 
Table 1 Potential mechanisms of milder COVID-19 in children

\begin{tabular}{ll}
\hline Hypothetical factors & Plausibile modes \\
\hline $\begin{array}{l}\text { 1. Immunological memory } \\
\text { 2. Viral interference }\end{array}$ & $\begin{array}{l}\text { Past exposure to common coronaviruses leads to cross-reaction antibodies formation } \\
\text { Existing colonization of nasal mucosa with ACE2 downregulating viruses interferes with SARS-CoV-2 repli- } \\
\text { cation } \\
\text { 3. Fewer co-morbidities }\end{array}$ \\
$\begin{array}{l}\text { Disease aggravating factors (e.g., heart disease, diabetes, arterial hypertension) are less common in children } \\
\text { 5. ACE2 and TMPRSS2 recep- } \\
\text { tors topography }\end{array}$ & $\begin{array}{l}\text { More effective innate immunity and less strong humoral immunity counteracts SARS-CoV-2 more successfully } \\
\text { Decreased expression in respiratory epithelium decreases viral attachment }\end{array}$ \\
\hline
\end{tabular}

TMPRSS2 transmembrane protease serine 2

$A C E 2$ angiotensin converting enzyme 2

Table 2 Potential mechanisms of milder COVID-19 in asthmatic children

\begin{tabular}{ll}
\hline Hypothetical factors & Plausible modes \\
\hline Th2-driven inflammation & IL-4, IL-5 and IL-13 lead to lower ACE2 expression in respiratory cells \\
Eosinophils & Reduction of viral load with subsequent attenuation of COVID-19 \\
$\begin{array}{l}\text { Inhaled corticosteroids as the } \\
\text { main treatment }\end{array}$ & $\begin{array}{c}\text { Downregulation of ACE2 and TMPRSS2 gene expression. Addition- } \\
\text { ally, IL-6 and IL-8 suppression }\end{array}$ \\
\hline
\end{tabular}

TMPRSS2 transmembrane protease serine 2

ACE2 angiotensin converting enzyme 2

Th2 T helper 2

IL interleukin
Moreover, type-2 inflammation seems to regulate the expression of ACE2 and TMPRSS2 in the nasal epithelia of asthmatic children [41]. Specifically, the Th2 response is mediated by IL-4, IL-5, and IL-13 [42]. These cytokines' action potentially reduces ACE2 in respiratory cells, leading to a better outcome in asthmatic children with COVID19. In isolation, IL-13 also acts on TMPRSS2, increasing airway mucus production. This countervailing effect from increased expression of TMPRSS2 is overridden by the synergistic effect of IL-4, IL-5, and IL-13 on ACE2 receptors. Furthermore, eosinophils (the end-product of IL-5 action on the bone marrow) seem to play a role in attenuating the COVID-19 burden in asthmatic children. Notably, low levels of eosinophils were observed in elderly patients who died of COVID-19 [41]. This seems to be supported by a large population-based cohort study, which reported that adults with non-allergic asthma had a higher risk of severe COVID19 than those with allergic asthma [43].

Additionally, a survey exploring data from 147 centers found 49 asthmatic children who tested positive for SARS$\mathrm{CoV}-2$. All children were admitted, but 29 required no treatment, 19 received supplemental oxygen, and only 4 required mechanical ventilation and admission to the pediatric intensive care unit (PICU). The authors speculated that the 29 untreated children were admitted purely for safety reasons. Regarding the 4 PICU admissions, the authors speculated that seasonal factors might have played a role. The study took place between March and April, when tree-pollen season had started, meaning pollen exposure could be the real reason for the PICU admissions with SARS-CoV-2 infection being coincidental [44]. Since the majority of asthmatic children belong to the allergic phenotype, this might explain their lower risk of severe COVID-19.

Another possible explanation of why the allergic asthma phenotype is protective against COVID-19 may be the drug that is mainly used to treat allergic asthmatics, namely steroids. The premise is that steroids may have a binary way of action against COVID-19. First, in a large cohort of well-characterized patients with asthma, inhaled steroid use was associated with lower sputum cell expression of ACE2 and TMPRSS2 [45]. Second, budesonide suppresses IL-6 and IL-8 production in vitro. Increased levels of IL-6 are a surrogate marker of worse outcomes in cases of severe COVID-19 pneumonia [46, 47].

However, despite downregulating cytokine production, budesonide failed to suppress the replication of SARS$\mathrm{CoV}-2$. This was achievable only with the use of ciclesonide and mometasone, which managed to halt viral replication in a manner comparable to lopinavir [48]. Thus, we could hypothesize that although the allergic phenotype is protective in young patients, it could be detrimental in elderly patients, leading to a severe disease course. More studies are needed to elucidate the role of Th2 response in children and adults with COVID-19. 


\section{Conclusion}

Clearly, our understanding of the relationship between SARS-CoV-2 infection and pediatric asthma is still explorative. Current data support a favorable outlook for asthmatic children infected by the new coronavirus. The way in which the innate and acquired immunity responds to the virus, a decreased number of virus host-receptors, and a downregulation of these receptors by Th2 inflammation and steroid treatment may all play roles in the way in which SARS-CoV-2 infects asthmatic children. Nevertheless, it is unclear whether we really have the whole picture. More studies are needed to clarify certain important details. For example, more knowledge is needed on whether the decreased number of admissions of asthmatic children with acquired COVID-19 is purely because of the mentioned features or whether there other contributing parameters.

During the quarantine period, we have shifted from one-to-one medical examination/consultation to telemedicine, schools have closed, and children have been taught how to maintain social distancing. We need to quantify the contribution of all these measures to the favorable outcome of asthmatic children infected by the virus. Papadopoulos et al. shed some light on this, but more studies are still needed. Moreover, we need to understand the way in which the virus behaves in asthmatic children treated with long-term oral steroids or one of the new biological drugs. Until the day when an effective vaccine and efficient treatment are available, we must follow the guidelines of the Centers for Disease Control to prevent viral spread, as well as those of the Global Initiative for Asthma (GINA) for treating asthmatic patients [49].

\section{Funding None.}

\section{Compliance with Ethical Standards}

Conflict of interest The author declares that they have no conflict of interest.

Consent for Publication Given by both the authors.

\section{References}

1. Guan WJ et al (2020) Clinical characteristics of coronavirus disease 2019 in China. N Engl J Med 382(18):1708-1720

2. Organization, W.H. WHO Director-general's opening remarks at the media briefing on COVID-19: 2020. https://www.who.int/dg/ speeches/detail/who-director-general-s-opening-remarks-at-theme dia-briefing-on-covid-19. Accessed 5 Jan 2020.
3. Li Y, Li H, Fan R, Wen B, Zhang J, Cao X et al (2016) Coronavirus infections in the central nervous system and respiratory tract show distinct features in hospitalized children. Intervirology 59:163-169. https://doi.org/10.1159/000453066

4. Guo Y, Zhou Y (2017) The detection and evaluation of pathogens and PCR methods for diagnosis of respiratory tract infection in children. Tian Med J 45:1005-1008. https://doi.org/10.11958 /20170856

5. Mallapaty S (2020) Why does the coronavirus spread so easily between people. Nature 579:183

6. Chan JF et al (2020) A familial cluster of pneumonia associated with the 2019 novel coronavirus indicating person-to-person transmission: a study of a family cluster. Lancet 2020:3957

7. Aaron B. Holley 2020 Does asthma lead to worse COVID-19 outcomes? Medscape (10223): 514-523

8. Corne JM, Marshall C, Smith S, Schreiber J, Sanderson G, Holgate ST et al (2002) Frequency, severity, and duration of rhinovirus infections in asthmatic and non-asthmatic individuals: a longitudinal cohort study. Lancet 359(9309):831-834

9. Jackson DJ, Trujillo-Torralbo MB, del-Rosario J, Bartlett NW NW, Edwards MR, Mallia P et al (2015) The influence of asthma control on the severity of virus-induced asthma exacerbations. J Allergy Clin Immunol 136(2):497-500

10. Sykes A, Edwards MR, Macintyre J, del Rosario A, Bakhsoliani E, Trujillo-Torralbo MB et al (2012) Rhinovirus 16-induced IFNalpha and IFN-beta are deficient in bronchoalveolar lavage cells in asthmatic patients. J Allergy Clin Immunol 129(6):1506-1514

11. Wark PA, Johnston SL, Bucchieri F, Powell R, Puddicombe S, Laza-Stanca V et al (2005) Asthmatic bronchial epithelial cells have a deficient innate immune response to infection with rhinovirus. J Exp Med 201(6):937-947

12. Contoli M, Message SD, Laza-Stanca V, Edwards MR, Wark PA, Bartlett NW et al (2006) Role of deficient type III interferon-lambda production in asthma exacerbations. Nat Med 12(9): 1023-1026

13. Jose A (2020) Castro-Rodriguez, Asthma and COVID-19 in children-a systematic review and call for data.

14. Lu X et al (2020) SARS-CoV-2 infection in children. N Engl J Med 382(17):1663-1665

15. Papadopoulos $\mathrm{N}$ et al (2020) Childhood asthma outcomes during the COVID-19 pandemic: findings from the PeARL multi-national cohort. medRxiv. https://doi.org/10.1101/2020.10.27.20219436

16. Papadopoulos $\mathrm{N}$ et al (2020) Impact of COVID-19 on pediatric asthma: Practice adjustments and disease burden. J Allergy Clin Immunol Pract 8:2592-2599. https://doi.org/10.1016/ jaip.2020.06.001

17. de Souza TH et al (2020) Clinical manifestations of children with COVID-19: a systematic review. Pediatr Pulmonol 55(8):18921899. https://doi.org/10.1002/ppul.24885

18. Yasuhara $\mathrm{J}$ et al (2020) Clinical characteristics of COVID-19 in children: a systemic review. Pediatr Pulmonol. https://doi. org/10.1002/ppul.24991

19. Prompetchara E, Ketloy C, Palaga T (2020) Immune responses in COVID-19 and potential vaccines: lessons learned from SARS and MERS epidemic. Asian Pac J Allergy Immunol 38(1):1-9

20. Imai Y, Kuba K, Penninger JM (2008) The discovery of angiotensin-converting enzyme 2 and its role in acute lung injury in mice. Exp Physiol 93:543-548

21. Verdecchia P, Cavallini C, Spanevello A, Angeli F (2020) The pivotal link between ACE2 deficiency and SARS-CoV-2 infection. Eur J Intern Med 76:14-20

22. Lanz TV et al (2010) Angiotensin II sustains brain inflammation in mice via TGF-beta. J Clin Invest 120:2782-2794

23. Bunyavanich S, Do A, Vicencio A (2020) Nasal gene expression of angiotensin-converting enzyme 2 in children and adults. JAMA $323: 2427-2429$ 
24. Wang A et al (2020) Single nucleus multiomic profiling reveals age-dynamic regulation of host genes associated with SARS-CoV-2 infection. bioRxiv. https://doi. org/10.1101/2020.04.12.037580

25. Muus $C$ et al (2020) Integrated analyses of single-cell atlases reveal age, gender, and smoking status associations with cell typespecific expression of mediators of SARS-CoV-2 viral entry and highlights inflammatory programs in putative target cells. Biorxiv. https://doi.org/10.1101/2020.04.19.049254

26. Dijkman R et al (2012) Replication-dependent downregulation of cellular angiotensin-converting enzyme 2 protein expression by human coronavirus NL63. J Gen Virol 93:1924-1929

27. Zimmermann P, Curtis N (2020) COVID-19 in children, pregnancy and neonates: A review of epidemiologic and clinical features. Pediatr Infect Dis J 39:469-477

28. Kumar N, Sharma S, Barua S, Tripathi BN, Rouse BT (2018) Virological and immunological outcomes of coinfections. Clin Microbiol Rev 31:e00111-e117

29. Li W et al (2003) Angiotensin-converting enzyme 2 is a functional receptor for the SARS coronavirus. Nature 426:450-454

30. Walls AC et al (2020) Structure, function, and antigenicity of the SARS-CoV-2 spike glycoprotein. Cell 181:281-292.e6

31. Schuler BA et al (2020) Vanderbilt COVID-19 consortium cohort; HCA lung biological network, age-related expression of SARSCoV-2 priming protease TMPRSS 2 in the developing lung. bioRxiv 2020.05.22.111187

32. Dong Y et al. (2020) Epidemiological characteristics of 2143 pediatric patients with 2019 coronavirus disease in China. Pediatrics

33. Viner RM, Whittaker E (2020) Kawasaki-like disease: Emerging complication during the COVID-19 pandemic. Lancet 395:17411743. https://doi.org/10.1016/S0140-6736(20)31129-6Medline

34. Feldstein LR et al (2020) Overcoming COVID-19 investigators; CDC COVID-19 response team, multisystem inflammatory syndrome in U.S. children and adolescents. N. Engl. J. Med. 383:334 346. https://doi.org/10.1056/NEJMoa2021680Medline

35. Pierce $C A$ et al (2020) Immune responses to SARS-CoV-2 infection in hospitalized pediatric and adult patients. Sci Transl Med. https://doi.org/10.1126/scitranslmed.abd5487

36. Zhou F, Yu T, Du R, et al. (2020) Clinical course and risk factors for mortality of adult inpatients with COVID-19 in Wuhan, China: a retrospective cohort study. Lancet

37. Williamson EJ, Walker AJ, Bhaskaran K et al (2020) Factors associated with COVID-19-related death using OpenSAFELY. Nature 584:430-436. https://doi.org/10.1038/s41586-020-2521-4
38. Pakkasela J, Ilmarinen P, Honkamäki J et al (2020) Age-specific incidence of allergic and non-allergic asthma. BMC Pulm Med 20:9. https://doi.org/10.1186/s12890-019-1040-2

39. Wu C et al (2020) Risk factors associated with acute respiratory distress syndrome and death in patients with coronavirus disease 2019 pneumonia in Wuhan, China. JAMA Intern Med. https://doi. org/10.1001/jamainternmed.2020.0994

40. Jackson DJ et al (2020) Association of respiratory allergy, asthma, and expression of the SARS-CoV-2 receptor ACE2. J Allergy Clin Immunol. https://doi.org/10.1016/j.jaci.2020.04.009

41. Sajuthi SP et al (2020) Type 2 and interferon inflammation strongly regulate SARS-CoV-2 related gene expression in the airway epithelium. bioRxiv. https://doi.org/10.1101/2020.04.09.034454

42. Steinman L (2007) A brief history of $\mathrm{T}(\mathrm{H}) 17$, the first major revision in the $\mathrm{T}(\mathrm{H}) 1 / \mathrm{T}(\mathrm{H}) 2$ hypothesis of $\mathrm{T}$ cell-mediated tissue damage. Nat Med 13:139-145

43. Zhaozhong Zhu et al. Association of asthma and its genetic predisposition with the risk of severeCOVID-19. J Allerg Clin Immunol 146(2)

44. Moeller A. et al. (2020) COVID-19 in children with underlying chronic respiratory diseases: survey results from 147 centers. ERJ open research. Article on review process

45. Peters MC (2020) COVID-19-related genes in sputum cells in asthma relationship to demographic features and corticosteroids. Am J Respir Crit Care Med 202(1):83-90. https://doi.org/10.1164/ rccm.202003-08210C

46. Yamaya M, Nishimura H, Deng X et al (2020) Inhibitory effects of glycopyrronium, formoterol, and budesonide on coronavirus HCoV-229E replication and cytokine production by primary cultures of human nasal and tracheal epithelial cells. Respir Investig 58(3): $155 \mathrm{e} 168$

47. Suda K, Tsuruta M, Eom J et al (2011) Acute lung injury induces cardiovascular dysfunction: effects of IL- 6 and budesonide/formoterol. Am J Respir Cell Mol Biol. 45(3):510e516

48. Matsuyama S, Kawase M, Nao N et al (2020) The inhaled corticosteroid ciclesonide blocks coronavirus RNA replication by targeting viral NSP15. bioRxiv. https://doi.org/10.1101/2020.03.11.987016

49. COVID-19: GINA answers to frequently asked questions on asthma management. 2020. https://ginasthma.org/covid-19-ginaanswers-tofrequently-asked-questions-on-asthma-management/.

Publisher's Note Springer Nature remains neutral with regard to jurisdictional claims in published maps and institutional affiliations. 